Original Paper http://ajol.info/index.php/ijbcs http://indexmedicus.afro.who.int

\title{
Fragmentation de l'espace et conflits d'usage au sahel : cas du bassin versant de Yakouta (Burkina Faso)
}

\author{
Blaise OUEDRAOGO*, Lucien OUEDRAOGO et Oumar KABORE \\ Institut de l'Environnement et de Recherches Agricoles (INERA), Cellule de Télédétection et d'Information \\ Géographique (CTIG) 01 BP 476 Ouagadougou, Burkina Faso. \\ *Auteur correspondant; E-mail : blaise32fr@yahoo.fr
}

\section{RESUME}

Face à la croissance de la population et au développement de l'agropastoralisme dans le bassin versant de Yakouta, l'occupation de l'espace connaît une forte modification donnant lieu à des conflits d'usage. L'objectif de cette recherche est d'analyser les mutations spatio-temporelles de l'occupation des terres dans le bassin versant de Yakouta et de ses conséquences sur les activités agropastorales. La méthodologie s'appuie sur des données de télédétection et d'enquêtes sur le terrain. L'étude diachronique de l'occupation des terres du bassin versant des années 2002 et 2011 révèle que les superficies des formations ripicoles ont connu une réduction de l'ordre de $-7,82 \%$ et une augmentation de $1,59 \%$ et de $15,71 \%$ respectivement pour les cultures et les plans d'eau. La modélisation de la dynamique de l'occupation des terres par la matrice de transition montre que les catégories en diminution concernent les steppes arbustive et arborée en faveur des champs de cultures. Les quantités de changements d'occupation de terre observés entre les deux dates est supérieure à $40 \%$ pour les steppes arbustives, $22 \%$ pour les cultures, et $1,76 \%$ pour les sols nus. Plus de 550 nouveaux ilots d'occupation sont apparus en 9 ans augmentant le niveau de fragmentation de l'espace avec une difficile cohabitation de l'élevage et de l'agriculture en saison pluvieuse.

(C) 2015 International Formulae Group. All rights reserved.

Mots clés: Activité agropastorale, bassin versant, fragmentation de l'espace, Sahel, Burkina Faso.

\section{Spatial fragmentation and usage conflicts in the Sahel: case of Yacouta catchment (Burkina Faso)}

\begin{abstract}
Under the rapid population growth and the increasing agro-pastoral activities, the land cover in the Yacouta catchment is experiencing strong changes leading to use conflicts. The objective of this study is to examine the spatio-temporal shift of land cover and its impacts on the agro-pastoral activities in the catchment. Remotely-sensed data over 9-year period were used to detect, analyze and model land cover change in the catchment. Results revealed that from 2002 to 2011, the area of gallery forest decreased with a rate of $7.8 \%$ while the areas of cropland and water body increased with the rates of $1.6 \%$ and $15.7 \%$, respectively. The transition matrix showed that the area of cropland is increasing at the expense of the shrub and wood steppes.
\end{abstract}


Within the two dates, the highest net change was observed for shrub steppe (40\%) followed by cropland (22\%) and barre land (1.7). About 550 new patches come out within the 9-year period highlighting the high degree of the fragmentation of the landscape. This fragmentation is source of conflicts due to high competition for space between farmers and breeders during rainy seasons.

(C) 2015 International Formulae Group. All rights reserved.

Keywords: Agro pastoralism, catchment, spatial fragmentation, Sahel, Burkina Faso.

\section{INTRODUCTION}

Face aux sécheresses à répétition qui ont marqué l'ensemble du Sahel, et à l'insuffisance de bonnes terres de culture, les producteurs agricoles se sont trouvés dans l'obligation d'occuper les terres autrefois marginales. L'agriculture et l'élevage constituent les principales activités dans le sahel burkinabè. La configuration du paysage y est dictée par les activités humaines en réponse aux besoins socioéconomiques des populations (Fahrig, 2003) et aussi par la variabilité climatique (Brandt et al., 2014).

Le développement de l'agropastoralisme au sahel a des conséquences sur les modes d'occupation et d'utilisation des sols (Aquino, 2000 ; Steffen et al., 2004). Les exploitations font face à un choix difficile entre les cultures et les animaux en ce qui concerne l'occupation de la terre pendant la saison des pluies (Bolwig et al., 2011). La fréquence des poches de sécheresse oblige les producteurs à un choix raisonné des terres cultivables; les bas-fonds et les bordures de plans d'eau autrefois réservés aux pâturages sont désormais destinés à l'agriculture. Les bas-fonds présentent, en effet, de nos jours, un potentiel très important de développement et d'intensification de la production agricole (Mameri et al., 2008). En raison de leur stock hydrique important, de leur richesse en matière organique et en azote dans l'horizon superficiel des sols argileux, ces milieux permettent de développer des cultures exigeantes en eau comme le riz ou à cycle plus long comme le sorgho. La mise en culture de ces zones déprimées est une réponse au manque d'eau en particulier en fin de cycle végétatif. Elles sont une dernière facette du paysage pour l'extension des surfaces cultivées (Chabi et al., 2010). L'intensité d'occupation des terres se traduisant le plus souvent par une fragmentation de l'espace suivie de dégradation des ressources naturelles (Barima et al., 2010 ; Gonin et Tallet, 2012; Kadeba et al, 2014). Mbow et al. (2015) certifient que la fragmentation modifie la taille, les formes et l'isolement des taches du paysage. Cet effritement de l'espace rend difficile la pratique de l'agriculture et de l'élevage qui n'ont pas d'espace spécifique, d'où des conflits d'usages (Bechir et al., 2015; Aubague, 2011). Kiema et al. (2015) ont montré que les dégâts des champs représentent $41 \%$ dans les difficultés de mobilités des animaux dans la zone d'étude. La question qui se pose est comment, concilier agriculture et élevage utilisant un même espace en pleine mutation? Pour baliser l'étude, l'hypothèse selon laquelle la fragmentation de l'espace est source de conflits dans la pratique de l'agriculture et de l'élevage a été émise. L'objectif étant d'analyser les mutations spatio-temporelles de l'espace et ses conséquences sur la pratique de l'agriculture et de l'élevage dans le bassin versant. 


\section{Le site de l'étude}

Le site d'étude est un bassin versant qui se situe dans la région sahélienne du Burkina Faso, entre $13^{\circ} 49^{\prime} 18,12^{\prime \prime}$ et $14^{\circ} 12^{\prime} 45,36^{\prime \prime}$ de latitude Nord et entre $0^{\circ} 36^{\prime} 30,96^{\prime \prime}$ et $0^{\circ} 4^{\prime} 55,2^{\prime \prime}$ de longitude Ouest (Figure 1). Ce bassin versant couvre une superficie d'environ 1800 $\mathrm{km}^{2}$ et regroupe trente-cinq villages administratifs répartis entre les communes de Dori à l'Est et de Gorgadji à l'Ouest. Le site de l'étude présente des caractéristiques spécifiques sur le plan climatique, physiographique, de végétation et de sols.

Sur le plan climatique, il est caractérisé par une pluviométrie faible et aléatoire. Avec une moyenne pluviométrique annuelle de 400 $\mathrm{mm}$ d'eau. Les températures sont très élevées avec une moyenne toujours supérieure à 30 ${ }^{\circ} \mathrm{C}$. L'évaporation est également très élevée et on estime à plus de $2 \mathrm{~m}$ d'eau évaporée chaque année (Ouédraogo, 2015). Au Sahel, la pluie est la variable climatique la plus déterminante pour la vie des populations. Certains auteurs considèrent qu'elle permet, à elle seule, de déterminer l'évolution de l'environnement dans cette région (Ali, 2010).

$\mathrm{Au}$ plan physiographique, on note la présence de cordons dunaires, de glacis, de mares et cours d'eau avec quelques buttes rocheuses ou cuirassées de type résiduel (Ouédraogo et al., 2013). Les formations végétales vont de la steppe arbustive à la steppe herbeuse. Elles sont remplacées aux abords des cours d'eau, par la forêt galerie à Hallea Inermis. C'est le domaine sahélien. Ces formations végétales sont très dégradées à cause des actions anthropiques notamment le surpâturage (Diallo, et al., 2011).

Les sols du bassin versant sont caractérisés par une grande hétérogénéité à cause de la simultanéité et de la succession de nombreux processus y compris le paléoclimat et le climat actuel, les dépôts dunaires de type éolien, l'érosion hydrique et éolienne, les remontées et les accumulations de sels, la formation de croûtes plasmiques, etc. (Thiombiano, 2000).

Les systèmes de production se résument à l'agriculture pluviale, à l'élevage avec une intégration progressive des deux activités désignée sous le vocable agropastoralisme. Les spéculations agricoles sont les céréales (mil, sorgho et maïs) et les oléo-protéagineux, (niébé, arachide et voandzou). L'élevage pratiqué au sahel burkinabè est de type pastoral, avec un cheptel estimé à plus de 1,76 million de bovins, 1,19 million d'ovins, 2,13 millions de caprins (DGPSE, 2012). Dans ce système d'élevage, l'alimentation des animaux provient essentiellement de l'exploitation des pâturages naturels par le biais de la conduite des troupeaux aux pâturages, et à la pratique de la transhumance. Ces pratiques traditionnelles actuelles ne permettent plus de répondre efficacement aux problèmes d'alimentation et d'abreuvement des animaux.

\section{MATERIEL ET METHODES}

\section{Collecte des données}

Pour atteindre les objectifs de cette étude, plusieurs outils et supports cartographiques ont été utilisés : des images satellites, un questionnaire, des logiciels de traitement des données et un Global positioning système (GPS).

- Deux images Landsat satellites TM (Thematic Mapper) du 14 octobre 2002 et ETM+ (Enhanced Thematic Mapper) du 2 février 2011 de la scène Path 194, Row 050 ont servi à la cartographie. Leur résolution spatiale est de 30 mètres, la projection est UTM 30 Nord avec l'ellipsoïde de référence WGS84. 
- Le questionnaire d'enquête a permis la collecte des données socio-économiques

- Les logiciels Arcgis10, Envi 4.8, Sphinx et Excel 2010 ont servi au traitement, à l'analyse et au calcul des indices de la dynamique structurale de l'espace.

- Le GPS Garmin CSx 60 a été à la base de levés de terrain pour la validation du traitement des images.

\section{Méthodologie}

La méthodologie utilisée s'est basée sur des techniques d'écologie du paysage à partir des deux images Landsat afin de caractériser la configuration spatiale du paysage par le calcul d'indices paysagers. Cette approche spatiale intègre les données d'enquête et des analyses à partir des Systèmes d'Information Géographiques (SIG).

\section{- Traitements des images satellites}

La connaissance du terrain a permis d'opter pour la classification supervisée pour identifier les classes d'occupation des terres du bassin versant de Yakouta en 2002 et 2011. Elle s'est basée sur l'algorithme dite de maximum de vraisemblance de Envi 4.8. Les canaux 4,3 et 2 ont été choisis en raison de la forte capacité de discrimination du canal 4 sensible à la végétation (Robin, 2002; Mas 2000). Cette classification a donné lieu à sept classes d'occupation: eau, steppe arborée, steppe arbustive, steppe herbeuse, sols nus, forêt galerie et champs. Pour éliminer les pixels isolés et rendre homogène les résultats de la classification en vue de la vectorisation, les résultats de traitement obtenus ont été filtrés. Envi® propose un filtre majoritaire et minoritaire appliqué sur une fenêtre de dimension $3 \times 3$ pixels. Le résultat de la classification a ensuite été vectorisé pour la cartographie dans Arc GIS. Ce processus a permis la segmentation de l'image, les unités s'individualisent et chacune d'elle peut être modifiée sans entraîner les autres.

\section{Données issues des enquêtes}

Les données d'enquêtes ont été dépouillées sous le logiciel Sphinx. Les résultats présentés sous forme de table et de graphiques ont permis de déterminer les tendances du développement des systèmes agricoles.

\section{Analyses statistiques}

Pour l'analyse de la structure de l'espace, plusieurs indices ont été calculés : l'aire des taches, le périmètre des taches, le taux moyen d'expansion spatiale et le changement par la matrice de transition

\section{- L'aire des taches (aj)}

$a j=\sum_{i=1}^{n} a i \quad$ Avec ai surface occupée par tache. L'augmentation du nombre de taches d'une classe peut être due à la fragmentation de cette classe (Davidson, 1998) ou à la création de nouvelles taches.

\section{- Le périmètre des taches $(\mathrm{P})$}

$P=\sum_{i=1}^{n} P i \quad$ Avec $P i$ le périmètre de la tache. La forme du périmètre est un indicateur de la régularité des surfaces.

\section{- Taux moyen d'expansion spatiale $(\mathbf{T})$}

La dynamique de l'occupation des terres s'explique par l'expansion spatiale des unités d'occupation qui peut être évaluée par l'une des formules appliquées pour mesurer la croissance des agrégats macroéconomiques entre deux périodes (Oloukoi et al., 2006). Le taux moyen d'expansion (T) est évalué à partir de la formule suivante:

$$
T=\frac{(\ln S 2-\ln S 1)}{t \ln e} \times 100
$$


Avec $\mathrm{t},=$ nombre d'années d'évolution; ln,=logarithme népérien: e,= base des logarithmes népériens e étant égale 2,71828. La variable considérée dans ce cas est la superficie (S). Ainsi, S1 et S2 correspondent respectivement à la superficie d'une unité d'occupation de l'année 2002 et de 2011.

\section{- Détection des changements par la}

\section{matrice de transition}

La matrice de transition est un tableau à double entrée qui permet de décrire, de manière condensée, les changements d'état des cellules d'occupation des terres intervenus entre deux dates données (Schlaepfer, 2002 cité par Oloukoi et al., 2006 ; Bamba et al., 2008).

Les valeurs des colonnes et des lignes correspondent à la proportion des aires occupée par chaque classe d'occupation de sol à la date correspondante. Les colonnes de la matrice indiquent les états d'occupation en 2011 ; les lignes correspondent aux états en 2002.

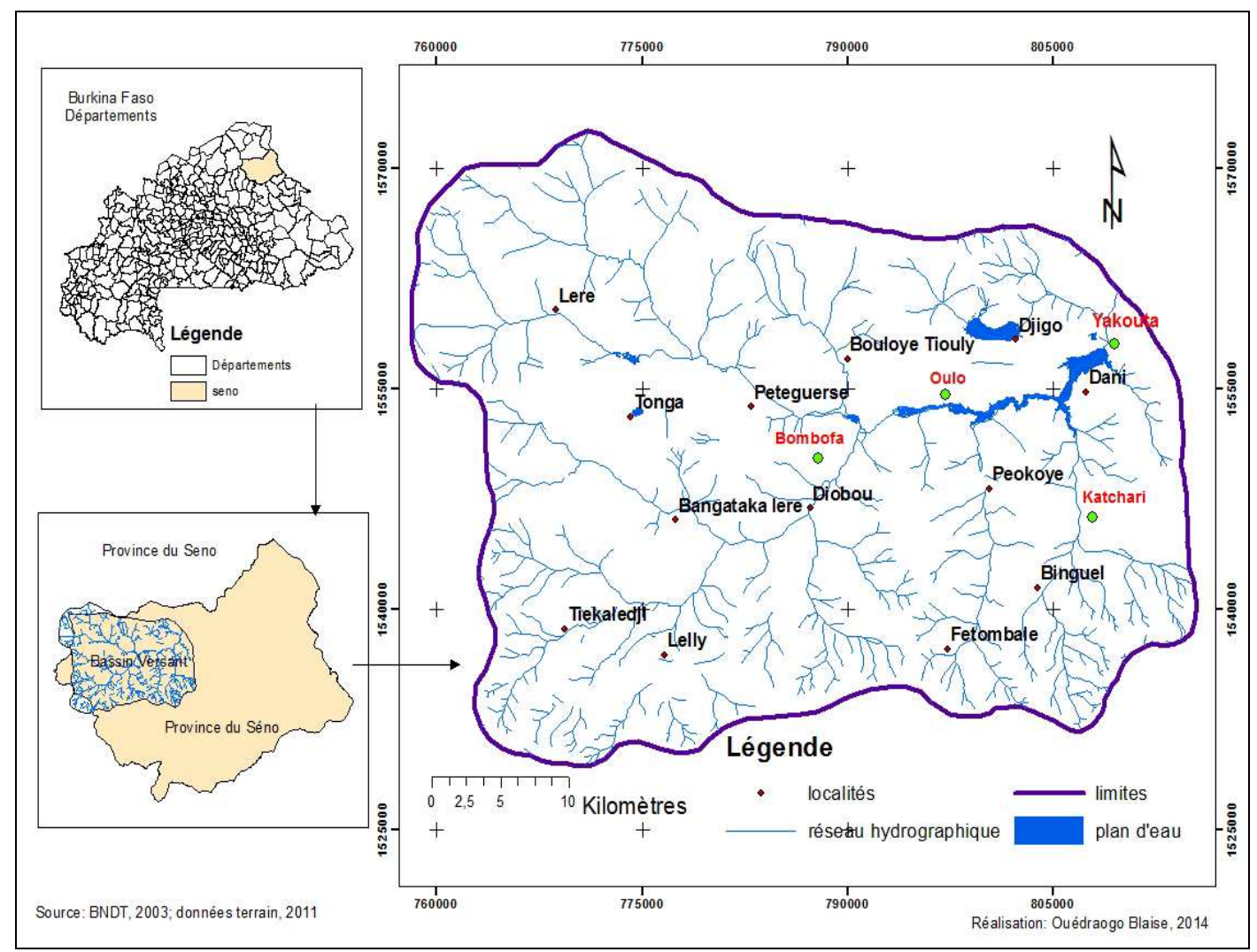

Figure 1: Localisation du secteur d'étude. 


\section{RESULTATS}

Précision des classifications des images Landsat de 2002 et 2011

La validité de la classification a été vérifiée par le calcul de quelques indices. Le « ROI separability » qui détermine le niveau de discrimination des sites d'entraînement a été calculé par la distance de JeffriesMatustita. Le résultat de cette séparabilité des régions d'entraînement a été supérieure à 1,9 . Les coefficients Kappa des classifications des images de 2002 et 2011 sont respectivement de 0,94 et 0,81 . Tous ces coefficients ont permis de justifier un bon niveau du traitement des images.

\section{Dynamique de l'occupation des terres}

Le traitement des images a permis de distinguer 7 types d'occupation des terres qui sont: les cultures (Cult), la formation ripicole (FR), la steppe arborée (Stp_Arbo), la steppe arbustive (Stp_Arbu), la steppe herbeuse (Stp_H), le sol nu (SN) et les plans d'eau (PE). L'analyse de la dynamique de l'occupation des terres a révélé une évolution des types d'occupation entre 2002 et 2011 dans le bassin versant de Yakouta.

L'occupation des terres avant l'installation du barrage est marquée par l'importance de la steppe arbustive (42\%) avec une occupation importante de l'agriculture $(28,2 \%)$ principalement sur les dunes de sable d'extension Est-Ouest du bassin versant de Yakouta. (Figure 2).

En 2011, après installation du barrage, on note une progression de l'agriculture $(32,53 \%)$ et une diminution de la steppe arbustive (42,2\%), (Figure 3).

La comparaison de l'évolution des unités d'occupation de l'année 2002 d'avec celles de 2011 montre la régression spatiale de plusieurs unités: steppe arbustive, steppe arborée, formation ripicole. Certaines unités, par contre, connaissent une augmentation comme les cultures, la steppe herbeuse et les sols nus. La dynamique de l'occupation des terres s'explique par l'expansion spatiale des unités d'occupation (Tableau 1).
On constate que l'unité «plan d'eau»a connu une forte dynamique $(+15 \%)$ et cela s'explique par la construction et la mise en eau du barrage en 2005. La régression remarquable de la formation ripicole $(-7,82 \%)$ est liée à la destruction de la végétation suite à la mise en place dudit barrage.

\section{Changements dans l'occupation des terres par la matrice de transition}

Le changement de l'occupation des terres a été évalué par la matrice de transition.

L'analyse synthétique des «transferts » d'occupation entre 2002 et 2011 permet de comprendre l'importance relative des dynamiques d'occupation des terres partitionnées et les transferts d'une unité à l'autre à partir du Tableau 2.

Ce tableau décrit les transferts des types d'occupation d'une classe à une autre. Ainsi par exemple, 16,37\% d'autres types d'occupation sont devenus des cultures et $5,17 \%$ des cultures sont devenus d'autres types d'occupation.

\section{Dynamique structurale de l'espace}

Les indices de paysage permettent d'évaluer le niveau de morcellement de l'espace et valident ou infirment les changements mis à jour dans la première méthode à savoir la détection des changements entre 2002 et 2011. Le nombre de taches est déterminé à partir de la table d'attributs de la carte de l'année considérée et fait ressortir le degré de fragmentation d'une classe entre deux périodes. Le Tableau 3 illustre cette fragmentation entre 2002 et 2011. La matrice de transition et les indices de structure spatiale ont montré le changement dans l'occupation des terres et l'émiettement de l'espace rendant difficile l'accès des animaux aux ressources (pâturage et eau). Le nombre de taches d'occupation des champs (Tableau 2) a augmenté de 552 ilots entre 2002 et 2011. 


\section{Conséquences sur les pratiques agricoles et} pastorales

La dynamique de l'occupation des terres notamment l'augmentation des superficies de cultures et leur effritement contribuent à une dégradation des ressources naturelles. Cette situation dans un contexte de variabilité climatique conduit inévitablement sur des conflits pour leur usage et leur appropriation. La recherche de terres favorables à l'implantation des champs a toujours eu pour corolaire une désapprobation des éleveurs qui y conduisaient leurs troupeaux. Les enquêtes ont montré qu'environ 3\% des agriculteurs ont délocalisé leurs champs pendant ces dix dernières années et $13,8 \%$ des enquêtés affirment occuper de nouvelles terres au niveau des bas-fonds autrefois destinés aux pâturages. Cette croissance du taux d'occupation pour l'agriculture (4\%) a des conséquences sur la végétation avec une disparition quasi-totale de la jachère dans le bassin versant de Yakouta. Pour l'élevage, la sédentarisation des populations nomades rend difficile la régénérescence du tapis végétal à cause de la pression constante des animaux sur le milieu. Le fait majeur de la fragmentation du paysage est la difficulté de pâturer les animaux pendant la saison pluvieuse. La conduite des animaux est rendue difficile par le morcellement des champs à forme variable et à taille réduite.

\section{Les types de conflits dans le bassin de Yakouta}

Les conflits d'usage de l'espace sont fonction des activités exercées. On retient principalement 3 types de conflits dans le bassin versant de Yakouta: les conflits liés à la gestion des animaux aux pâturages, les conflits liés au foncier et les conflits liés à la gestion des points d'eau.

- Les résultats des enquêtes ont montré que $43 \%$ de l'échantillon a subi des dégâts de cultures causés par les animaux pendant la saison pluvieuse et $32 \%$ des jardins maraîchers ont été touchés. En saison sèche en effet, les cultures maraîchères sont pratiquées par quelques producteurs, généralement venus d'autres régions. Les sites d'exploitation se situent au niveau des villages riverains du barrage (Yakouta, Dani, Oulo, Dangade et Peokoye) et exposés aux animaux venant s'abreuver.

- Les conflits liés à la propriété foncière représentent $13 \%$ de l'échantillon enquêté. Il s'agit généralement de litiges liés à la délimitation des champs et qui trouvent le plus souvent une solution auprès du chef de village.

- Les conflits nés autour des points (forage, puisards) ont lieu généralement en saison sèche et surtout dans les villages éloignés des plans d'eau (Lelly, Fetombalé, Bangre, Nakou, Malbo, Binguel). L'abreuvement des animaux est le point d'achoppement entre les bergers et les femmes venant puiser de l'eau pour le ménage ou même entre bergers.

Tableau 1: Expansion des unités d'occupation de terres entre 2002 et 2011.

\begin{tabular}{lc}
\hline Unités & Taux moyen d'expansion (\%) \\
\hline Cultures & 1,59 \\
formation ripicole & $-7,82$ \\
steppe arborée & $-2,06$ \\
steppe arbustive & $-1,27$ \\
steppe herbeuse & 1,55 \\
sol nu & 3,46 \\
plan d'eau & 15,71 \\
\hline
\end{tabular}


B. OUEDRAOGO et al. / Int. J. Biol. Chem. Sci. 9(6): 2727-2739, 2015

Tableau 2: Matrice de transition des classes d'occupation des terres en \% entre 2002 et 2011.

\begin{tabular}{|c|c|c|c|c|c|c|c|c|c|}
\hline \multirow[t]{2}{*}{2002} & \multicolumn{9}{|c|}{2011} \\
\hline & Cultures & $\begin{array}{c}\text { Formation } \\
\text { ripicole }\end{array}$ & $\begin{array}{c}\text { Plan } \\
\text { d'eau }\end{array}$ & $\begin{array}{c}\text { Sol } \\
\text { nu }\end{array}$ & $\begin{array}{c}\text { Steppe } \\
\text { arborée }\end{array}$ & $\begin{array}{c}\text { Steppe } \\
\text { arbustive }\end{array}$ & $\begin{array}{c}\text { Steppe } \\
\text { herbeuse }\end{array}$ & $\begin{array}{l}\text { Total } \\
2002 \\
\end{array}$ & Pertes \\
\hline Cultures & 18,24 & 3 & 0,4 & 1,07 & 0 & 0 & 0,7 & 23,41 & 5,17 \\
\hline formation ripicole & 1,22 & 3,29 & 0,31 & 0,04 & 0,18 & 0,29 & 0,27 & 5,6 & 2,31 \\
\hline plan d'eau & 0 & 0 & $\mathbf{0 , 0 7}$ & 0 & 0 & 0 & 0 & 0,07 & 0 \\
\hline sol nu & 0,12 & 0 & 0 & 1,48 & 0 & 0,01 & 0,22 & 1,83 & 0,35 \\
\hline steppe arborée & 1,95 & 3,37 & 0,11 & 0,14 & $\mathbf{0 , 1 1}$ & 1,79 & 1,98 & 9,45 & 9,34 \\
\hline steppe arbustive & 7,94 & 4,2 & 0,07 & 3,06 & 0 & 20,97 & 9,81 & 46,05 & 25,08 \\
\hline steppe herbeuse & 5,14 & 0 & 0,02 & 3,84 & 0 & 0,05 & 4,54 & 13,59 & 9,05 \\
\hline Total 2011 & 34,61 & 13,86 & 0,98 & 9,63 & 0,29 & 23,11 & 17,52 & 100 & \\
\hline Gain & 16,37 & 10,57 & 0,91 & 8,15 & 0,18 & 2,14 & 12,98 & & \\
\hline
\end{tabular}

Tableau 3: Fragmentation des unités d'occupation entre 2002 et 2011.

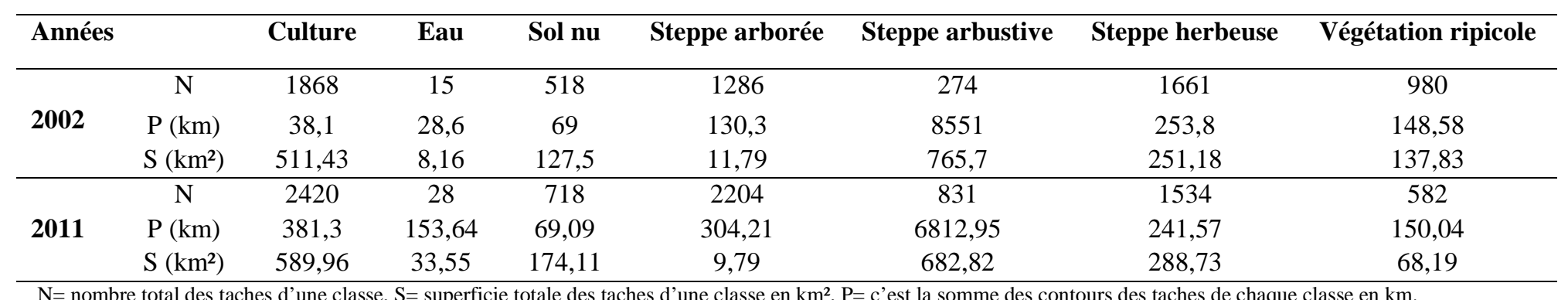

$\mathrm{N}=$ nombre total des taches d'une classe, $\mathrm{S}=$ superficie totale des taches d'une classe en $\mathrm{km}^{2}, \mathrm{P}=\mathrm{c}$ 'est la somme des contours des taches de chaque classe en $\mathrm{km}$. 


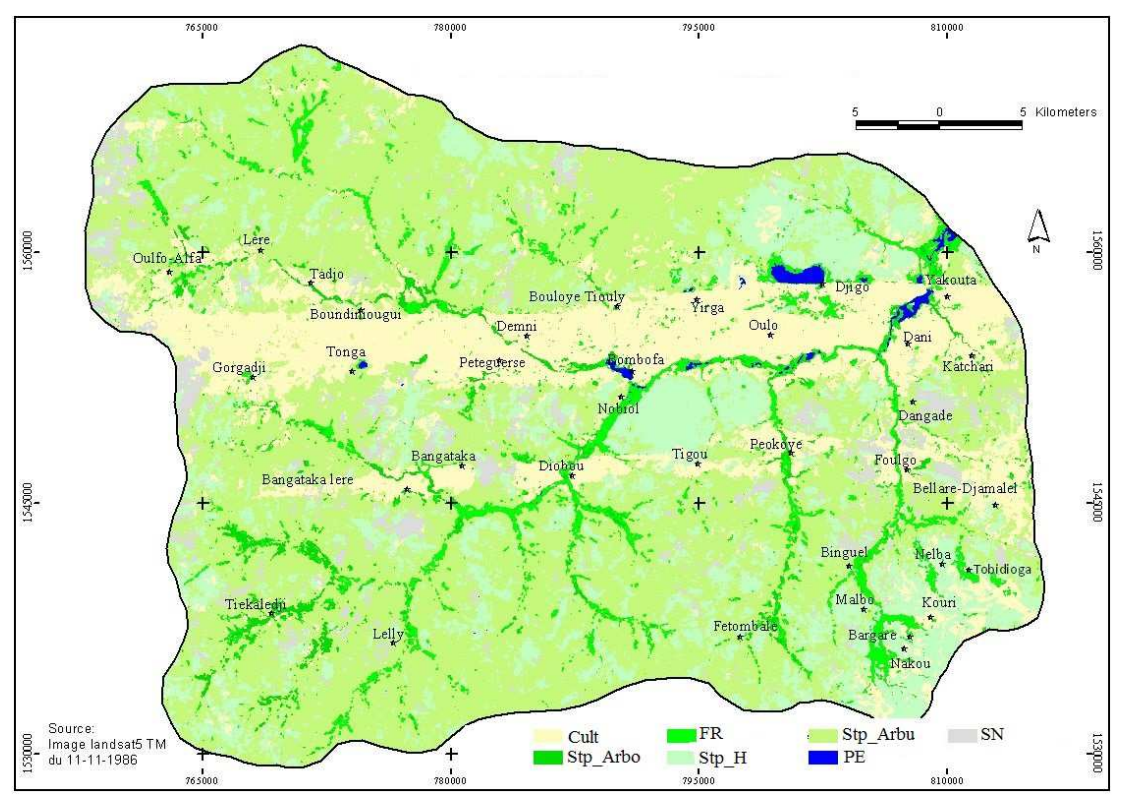

Figure 2: Occupation des terres en 2002. Cultures = Cult ; Formation ripicole = FR ; Steppe arborée = Stp_Arbo ; Steppe arbustive $=$ Stp_Arbu $;$ Steppe herbeuse $=$ Stp_H Sol nu=SN $;$ Plan d'eau $=$ PE.

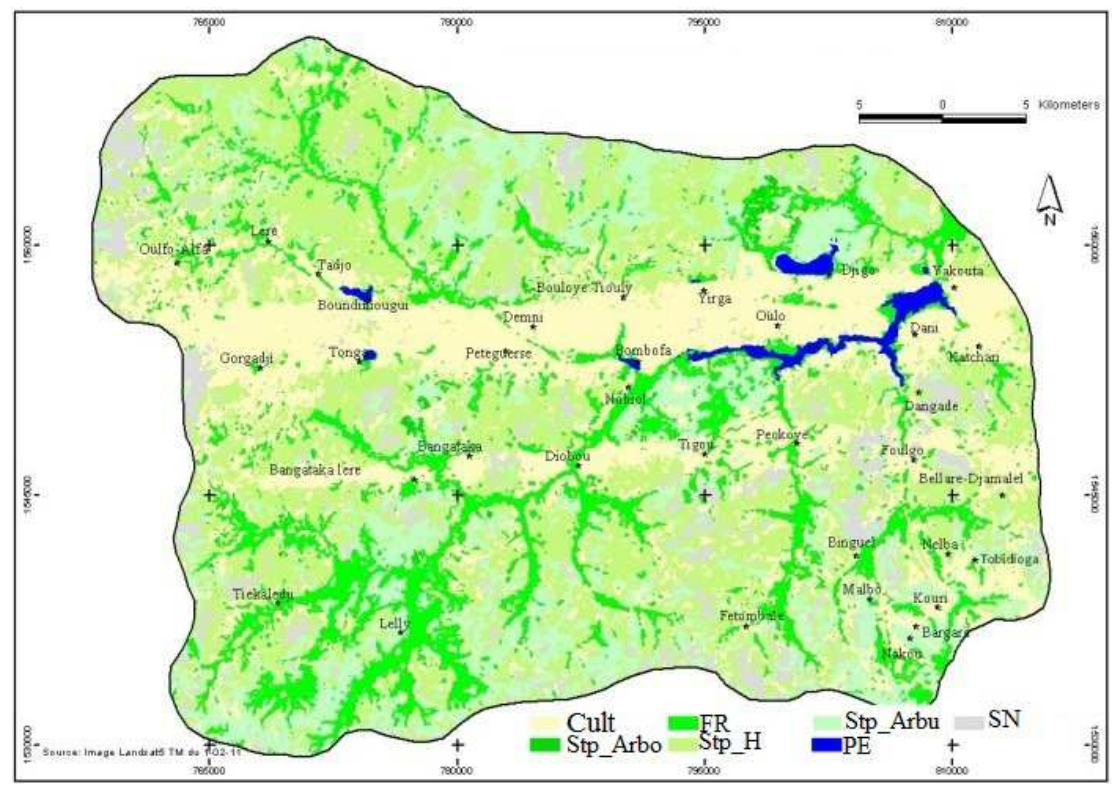

Figure 3 : Occupation des terres en 2011. Cultures $=$ Cult ; Formation ripicole $=$ FR; Steppe arborée $=$ Stp_Arbo $;$ Steppe arbustive $=$ Stp_Arbu $;$ Steppe herbeuse $=$ Stp_H Sol nu=SN $;$ Plan d'eau $=$ PE 


\section{DISCUSSION}

Le secteur d'étude est situé dans le sahel burkinabé où les conditions climatiques sont défavorables avec 3 mois de pluie et d'une précipitation moyenne de $400 \mathrm{~m} / \mathrm{an}$. C'est dans ces conditions que le barrage de Yakouta construit en 2005 a favorisé le développement de l'agropastoralisme et avec une forte occupation des terres. Le choix du site s'explique donc par l'importance des activités agropastorales et l'afflux des populations migrantes vers la zone d'étude. Les images Landsat TM bien que la résolution soit assez faible $(30 \mathrm{~m})$ ont permis d'identifier les catégories d'occupation des terres. La taille du pixel $30 \mathrm{~m}$ x $30 \mathrm{~m}$ n'a pas permis d'accéder à des détails. A l'avenir, afin d'obtenir des résultats plus précis et détaillés, des images hautes résolutions $(5 \mathrm{~m} \times 5 \mathrm{~m}$ au moins) seront nécessaires. Les résultats de cette étude trouvent ses limites dans la résolution spatiale de l'image utilisée mais permettent suffisamment de mesurer la tendance de la dynamique de l'occupation des terres.

Ces résultats obtenus confirment les travaux de plusieurs auteurs sur l'impact de l'anthropisation sur le paysage (Oloukoï et al., 2006; Bamba et al., 2010 ; Diallo et al., 2011; Inoussa et al., 2011; Hiernaux et al., 2014). Dans la plupart des cas, l'agriculture est responsable des répercussions directes sur l'occupation des terres et la configuration du paysage. L'anthropisation du milieu naturel étant caractérisée par la fragmentation. Mama et al. (2013) ont montré que la fragmentation se traduit par un changement dans la géométrie des taches et une évolution des classes anthropisées au détriment des classes naturelles d'occupation du sol. En effet, l'augmentation du nombre des taches est significative (552 ilots en moins de 10 ans), ce qui montre un morcellement important des terres agricoles. L'effritement de l'espace pose donc un problème d'aménagement du territoire. Les conflits d'usage de l'espace se déroulent entre acteurs concernés par le problème d'appropriation du lieu (Taibi et al., 2009). Torre et al. (2010) tirent la sonnette d'alarme quand ils disent que quelle que soit la situation de départ, les conflits peuvent connaître des expansions sociales et spatiales, dès lors qu'ils cristallisent un ensemble d'enjeux à dimension sociétale. Plus l'espace se raréfie, plus les tensions sont importantes. Elodie (2013) pose le problème de la délimitation de l'espace à vocation pastorale comme préalable pour limiter les conflits. Il est essentiel de clarifier le droit aux ressources, d'établir une sorte de cadastre où chaque parcelle est répertoriée avec le nom de son propriétaire, et de réglementer, au niveau local, le mode d'accès aux pâturages qui demeure imprécis. Ainsi, les conflits entre les utilisateurs des ressources devraient diminuer et la sécurité territoriale favoriser l'investissement. Cette vision peut-elle être viable au sahel où les ressources végétales sont limitées et avec des populations à traditions nomades? Dans le contexte du site d'étude, le caractère désertique et de dunes de sables rendent la partie utile du bassin versant très limitée donc difficile à un zonage. Cette étude révèle aussi l'importance de la modélisation par la matrice de transition, dans la compréhension de la dynamique du paysage.

\section{Conclusion}

Les résultats obtenus montrent une croissance des activités agropastorales dans le site de l'étude. Le manque de zonage spatial et l'empiétement des zones autrefois dédiées au pâturage rendent difficile la pratique de l'élevage transhumant. Dans un contexte d'intégration agriculture-élevage adoptée par les populations depuis les dernières sécheresses, les conflits d'usage de l'espace font désormais partie intégrante des systèmes agropastoraux. Les instances traditionnelles de résolution des conflits liés aux dégâts des champs par les animaux sont perturbées avec 
l'introduction des nouvelles règles de gestion des conflits par l'administration moderne. Les parties prenantes sont le plus souvent tentées de se rendre justice avec des conséquences énormes sur la vie des populations. De nos jours, les conflits sont moins nombreux et trouvent généralement des solutions auprès du chef de village. Mais au-delà de cette conclusion, des investigations avec des considérations sociodémographiques pourraient aider à comprendre davantage les raisons essentielles de cette apparente harmonie.

\section{CONFLIT D'INTÉRÊT}

Les auteurs déclarent qu'il n'existe aucun conflit d'intérêt pour cet article.

\section{CONTRIBUTIONS DES AUTEURS}

BO a collecté les données, traité les images satellites, a realisé l'analyse diachronique de l'occupation des terres et a rédigé le manuscrit; $\mathrm{LO}$ a dépouillé les données d'enquêtes, analysé spatiale et a participé à la relecture du manuscrit; OK a analysé des données d'enquête, a participé à la rédaction et la relecture du manuscrit.

\section{REFERENCES}

Ali A. 2010. La variabilité et les changements climatiques au Sahel. Comprendre la situation actuelle par l'observation. In Le Sahel face aux changements climatiques. Enjeux pour un développement durable. Bulletin Mensuel, Centre Régional Agrhymet, Niamey, Numéro spécial, pp. 17-20.

Aubague S, Mannany AA, Grimaud P. 2011. Difficultés de transhumance des chameliers dans le Tchad central liées aux aléas climatiques. Sécheresse 22(1): 25-32. DOI : 10.1684/sec.2011.0295.

Bamba I, Barima YSS, Bogaert J. 2010. Influence de la densité de la population sur la structure spatiale d'un paysage forestier dans le bassin du Congo en R.D. Congo. Tropical Conservation Science, 3(1): 31-44. DOI : urn:issn:1940-0829

Barima YS, Egnankou MW, N'doume CTA, Kouame FN, Bogaert J. 2010. Modélisation de la dynamique du paysage forestier dans la région de transition forêt- savane à l'Est de la Côte d'Ivoire. Revue de Télédétection, 9(2): 129-138. http://hdl.handle.net/ 2268/106380.

Bechir AB, Logtene YM. 2015 Analyse de la végétation pâturée autour des ouvrages hydrauliques dans le Département du Batha Est au Tchad. Int. J. Biol. Chem. Sci., 9(3): 1557-1570, DOI : http://dx.doi.org/10.4314/ijbcs.v9i3.36

Bolwig S, Rasmussen K, Hesse C, Hilhorst T, Hansen MK. 2011. New Perspectives on Natural Resource Management in the Sahel; SEREIN Occasional Paper; Department of Geography and Geology, University of Copenhagen: Copenhagen, Denmark.

Brandt M, Grau T, Mbow C. and Cyrus Samimi 2014: Modeling Soil and Woody Vegetation in the Senegalese Sahel in the Context of Environmental Change. Land, 3: 770-792; DOI : 10.3390/land3030770

Chabi A, Oloukoi J, Mama V, Kiepe P. 2010. Inventaire par télédétection des agroécosystèmes de bas-fonds dans le centre du Bénin. Cahiers Agricultures, 19(6) : 446-453 ; DOI : 10.1684/agr.2010.0434, p. 446-453.

DGPSE (Direction générale de la prévision, des statistiques et de l'économie de l'élevage). 2012. Statistiques du sous secteur de l'élevage, Ouagadougou, Ministère des Ressources Animales, Ouagadougou, Burkina Faso, 155 p.

Diallo A, Ngom Faye M, Ndiaye O. Guisse A. 2011. Variations de la composition de la végétation herbacée des plantations 
de Acacia senegal (L.) Willd de la zone de Dahra (Ferlo). Int. J. Biol. Chem. Sci., $\quad$ 5(3): $\quad 1250-1264$, http://dx.doi.org/10.4314/ijbcs.v5i3.722 73

Diallo H, Bamba I, Barima YSS, Visser M, Ballo A, Mama A, Vranken I, Maiga M, Bogaert J. 2011. Effets combinés du climat et des pressions anthropiques sur la dynamique évolutive de la dégradation d'une aire protégée du Mali (la Réserve de Fina, Boucle du Baoulé). Sécheresse, 22(2): 97-107. DOI : $10.1684 / \mathrm{sec} .2011 .0306$

Élodie R. 2013. Les zones pastorales comme solution aux conflits agriculteurs / pasteurs au Burkina Faso : l'exemple de la zone pastorale de la Doubégué. Les Cahiers d'Outre-Mer, 249(63): 4771. DOI : $10.4000 / \mathrm{com} .5861$

Fahrig L. 2003. Effects of habitat fragmentation on biodiversity. Annual Review of Ecology, Evolution and Systematics, 34: 487-515. DOI: 10.1146/annurev.ecolsys.34.011802.13 2419

Gonin A, Tallet B. 2012. Changements spatiaux et pratiques pastorales : les nouvelles voies de la transhumance dans l'Ouest du Burkina Faso. Cahiers Agricultures, 21(6): 448-454. DOI:10.1684/agr.2012.0595

Hiernaux P, Diawara M, Gangneron F. 2014. Quelle accessibilité aux ressources pastorales du Sahel ? L'élevage face aux variations climatiques et aux évolutions des sociétés sahéliennes, Revue Afrique Contemporaine, 1(249) : 21-35 DOI : 10.3917/afco.249.0021

Kadeba A, Sambare O, Soulama S, Thiombiano A, Schmidt M, Boussim JI. 2014. Typologie spatiale de la végétation sahélienne en relation avec les indicateurs de dégradation au Burkina Faso. Int. J. Biol. Chem.
Sci., 8(3): $\quad$ 1049-1064. DOI: http://dx.doi.org/10.4314/ijbcs.v8i3.19

Kiema A, Sawadogo I, Ouedraogo T, Nianogo AJ. 2012. Stratégies d'exploitation du fourrage par les éleveurs de la zone sahélienne du Burkina Faso. Int. J. Biol. Chem. Sci., 6(4): 1492-1505, DOI : http://dx.doi.org/10.4314/ijbcs.v6i4.8

Kiema A, Tontibomma GB, Zampaligré N. 2015. Transhumance et gestion des ressources naturelles au Sahel: contraintes et perspectives face aux mutations des systèmes de productions pastorales. VertigO-la Revue Electronique en Sciences de l'Environnement, 14(3). DOI : 10.4000/vertigo. 15404

Mama A, Sinsin B, De Cannière C, Bogaert J. 2013. Anthropisation et dynamique des paysages en zone soudanienne au nord du Bénin. Tropicultura, 31: 78-88. http://hdl.handle.net/2268/160259

Maméri C, Mohamed K, Miézan MK. 2008. Intensification de la riziculture de basfonds dans le Sine-Saloum (Sénégal). Cahiers Agricultures 17: 451- 5 DOI : 10.1684/agr.2008.0205

Mas JF. 2000. Une revue de méthodes et des techniques de télédétection du changement. Canadian Journal of Remote Sensing 26: 349-362 DOI:10.1080/07038992.2000.1087478 5

Mbow C, Brandt M, Ouedraogo I, Jan de Leeuw, Marshall M. 2015. What Four Decades of Earth Observation Tell Us about Land Degradation in the Sahel? Remote Sensing, 7: 4048-4067; DOI : 10.3390/rs70404048

Oszwald J, Lefebvre A, Arnauld De Sartre X, Thalès M, Gond V. 2010. Analyse des directions de changement des états de surface végétaux pour renseigner la dynamique du front pionnier de Maçaranduba (Para Bresil) entre 1997 
et 2006, 2010. Revue Télédétection, 9(2): 97-111<hal-00623686>

Ouédraogo B. 2015. Stratégies d'adaptation des agropasteurs à la variabilité climatique dans le bassin versant de Yakouta (Burkina Faso). Thèse de doctorat unique de l'Université de Ouagadougou, p.248.

Ouédraogo I, Savadogo P, Tigabu M, Dayamba SD, Oden PC. 2011. Systematic and random transitions of land-cover types in Burkina Faso, West Africa. International Journal of Remote Sensing. http://dx.doi.org/10.1080/ 01431161.2010.495095

Ouédraogo L, Ouédraogo B, Kaboré O, Yanogo PI, Zoungrana TP, Bouzou Moussa I. 2013. Localisation des zones d'accès à l'eau dans le bassin versant du Goudébo (Région de Yakouta, Burkina Faso). Physio-Géo Géographie Physique et Environnement, volume VII. DOI : 10.4000/physio-geo.3374

Oulokoi J, Mama VJ, Agbo FB. 2006. Modélisation de la dynamique de l'occupation des terres dans le Département des collines au Benin. Revue Télédétection, 6(4): 305-323.

Reenberg A, Maman I, Bouzou Moussa I, Fog B. 2013. Land Saturation in SE Niger:
Triangulating Qualitative and Quantitative Information for Critical Assessment of Land Use Trajectories. Land, 2: 508-533, doi: 10.3390/land2030508

Robin M. 2002. Télédétection, des satellites au SIG. Une analyse complète du processus de création d'un type essentiel d'information géographique, Nathan University, p.318.

Taibi AN, Barry MH, Senghor MW, Ballouche A, Moguedet G. 2009. Le bas delta du fleuve Sénégal face aux risques de dégradation et conflits d'usage dans un contexte de restauration des écosystèmes et des activités. Approche par les outils d'analyse spatiale. Journées d'Animation Scientifique (JAS09) Alger, Algérie.

Torre A, Melot R, Bossuet L, Cadoret A, Caron A, Darly S, Jeanneaux P, Kirat T et Vu Pham H, 2010. Comment évaluer et mesurer la conflictualité liée aux usages de l'espace ? Eléments de méthode et de repérage, VertigO - la Revue Electronique en Sciences de l'environnement, 10(1). DOI : 10.4000/vertigo.9590 